\title{
Kinetic and Structural Characterization for Cofactor Preference of Succinic Semialdehyde Dehydrogenase from Streptococcus pyogenes
}

\author{
Eun Hyuk Jang ${ }^{1}$, Seong Ah Park ${ }^{2}$, Young Min $\mathrm{Chi}^{{ }^{1, *}}$, and Ki Seog Lee ${ }^{2, *}$
}

\begin{abstract}
The $\gamma$-Aminobutyric acid (GABA) that is found in prokaryotic and eukaryotic organisms has been used in various ways as a signaling molecule or a significant component generating metabolic energy under conditions of nutrient limitation or stress, through GABA catabolism. Succinic semialdehyde dehydrogenase (SSADH) catalyzes the oxidation of succinic semialdehyde to succinic acid in the final step of GABA catabolism. Here, we report the catalytic properties and two crystal structures of SSADH from Streptococcus pyogenes (SpSSADH) regarding its cofactor preference. Kinetic analysis showed that SpSSADH prefers NADP ${ }^{+}$over NAD $^{+}$as a hydride acceptor. Moreover, the structures of SpSSADH were determined in an apo-form and in a binary complex with $\mathrm{NADP}^{+}$at $1.6 \AA$ and $2.1 \AA \AA$ resolutions, respectively. Both structures of SpSSADH showed dimeric conformation, containing a single cysteine residue in the catalytic loop of each subunit. Further structural analysis and sequence comparison of SpSSADH with other SSADHs revealed that Ser158 and Tyr188 in SpSSADH participate in the stabilization of the 2'-phosphate group of adenine-side ribose in $\mathrm{NADP}^{+}$. Our results provide structural insights into the cofactor preference of SpSSADH as the gram-positive bacterial SSADH.
\end{abstract}

\section{INTRODUCTION}

Bacteria and other microorganisms are exposed to various stressful conditions, including nutrient limitation or physical stress, in their natural environment. In such conditions, many of bacteria tend to convert into the stationary phase or dormant state in order to endure long-term nutrient limitation and starva-

${ }^{1}$ Department of Biosystems and Biotechnology, College of Life Sciences and Biotechnology, Korea University, Seoul 136-713, Korea, ${ }^{2}$ Department of Clinical Laboratory Science, College of Health Sciences, Catholic University of Pusan, Busan 609-757, Korea

*Correspondence: kslee@cup.ac.kr (KSL); ezeg@korea.ac.kr (YMC)

Received 10 June, 2014; revised 29 August, 2014; accepted 2 September, 2014; published online 26 September, 2014

Keywords: $\gamma$-aminobutyric acid (GABA) catabolism, cofactor preference, GabD, Streptococcus pyogenes, succinic semialdehyde dehydrogenase tion (Trainor et al., 1999; Wood et al., 2009). However, other bacteria that have not developed starvation-resistance require a supply of energy for survival, obtained by amino acid utilization and progressive protein synthesis (Trainor et al., 1999). The $\gamma$ Aminobutyric acid (GABA) is synthesized from glutamate by glutamate decarboxylase, and is a non-protein amino acid found in virtually all prokaryotic and eukaryotic organisms (Bouche and Fromm, 2004). GABA is used for various roles, including as a signaling molecule or as a significant component for generating metabolic energy under conditions of nutrient limitation or stress (Fait et al., 2008). GABA catabolism involves a two-step pathway, and the product is introduced into the tricarboxylic acid (TCA) cycle in the form of succinic acid (SA). In the first step of GABA catabolism, GABA transaminase converts GABA to succinic semialdehyde (SSA). Then, SSA is oxidized by succinic semialdehyde dehydrogenase (SSADH) to SA in the presence of $\mathrm{NAD}(\mathrm{P})^{+}$(Schneider et al., 2002).

SSADH, which is the enzyme in the final step of GABA catabolism, belongs to the aldehyde dehydrogenases (ALDHs) superfamily, and has strict substrate specificity that uses only SSA as the substrate (de Carvalho et al., 2011). Deficiency of SSADH causes rare autosomal recessive disease in humans, and similarly, causes various developmental and phenotypic changes in plants (Bouche et al., 2003; de Carvalho et al., 2011; Kim et al., 2011). In bacteria, SSADHs play important roles in metabolism, including in the detoxification of accumulated SSA, and survival under limited nutrient conditions (Fuhrer et al., 2007; Schneider et al., 2002). Escherichia coli has two genes, gabD and ynel (also called sad), encoding different SSADHs. While the gabD gene encodes an $\mathrm{NADP}^{+}$-dependent SSADH (GabD), the SSADH encoded by the ynel gene (Ynel) can use either NAD ${ }^{+}$ or NADP ${ }^{+}$as a cofactor (Fuhrer et al., 2007; Zheng et al., 2013).

The gram-positive human pathogen Streptococcus pyogenes causes numerous diseases ranging from mild upper respiratory tract and skin infections (e.g. pharyngitis and pyoderma) to extremely severe infections (e.g. streptococcal toxic syndrome and necrotizing fasciitis) (Cunningham, 2000; Ralph and Carapetis, 2013). S. pyogenes shows characteristics of persistent infection, such as asymptomatic carriage and failure of antibiotic treatment (Grant and Hung, 2013; Wood et al., 2009). During persistent infection, S. pyogenes adapts to a variety of environmental conditions by expression changes in the growth phase-associated genes (Chaussee et al., 2004; 2008; Wood et al., 2005). These 
Kinetic and Structural Studies on the Cofactor Preference of SpSSADH

Eun Hyuk Jang et al.

Table 1. Data collection and refinement statistics of SpSSADH

\begin{tabular}{|c|c|c|}
\hline PDB ID & $\begin{array}{c}\text { Apo } \\
\text { 4OGD }\end{array}$ & $\begin{array}{c}\text { NADP }^{+} \text {complex } \\
4 \mathrm{OHT}\end{array}$ \\
\hline \multicolumn{3}{|l|}{ Data collection } \\
\hline Space group & $P 2{ }_{1} 2_{1} 2_{1}$ & $P 4_{3} 2{ }_{1} 2$ \\
\hline Wavelength $(\AA)$ & 1.0000 & 0.97951 \\
\hline Cell dimensions $(\AA)$ & $a=93.26, b=100.28, c=105.10$ & $a=134.76, b=134.76, c=173.53$ \\
\hline Resolution range $(\AA)$ & $50.0-1.6(1.63-1.60)$ & $50.0-2.1(2.18-2.10)$ \\
\hline Total reflections & $1,262,499$ & 837,575 \\
\hline Unique reflections & 128,443 & 93,776 \\
\hline Completeness (\%) & $98.5(88.4)$ & $99.4(98.7)$ \\
\hline$R_{\text {merge }}^{a}(\%)$ & $7.1(30.6)$ & $12.6(44.1)$ \\
\hline Redundancy & $9.8(4.8)$ & $8.9(5.9)$ \\
\hline$\| / \sigma(I)$ & $26.3(2.5)$ & $21.5(3.7)$ \\
\hline \multicolumn{3}{|l|}{ Refinement } \\
\hline Resolution range $(\AA ̊)$ & $18.0-1.6$ & $39.9-2.1$ \\
\hline$R / R_{\text {free }}^{\mathrm{b}}(\%)$ & $17.4 / 19.9$ & $20.1 / 22.4$ \\
\hline \multicolumn{3}{|l|}{ No. of atoms } \\
\hline Protein & 7,148 & 7,148 \\
\hline Water & 527 & 614 \\
\hline $\mathrm{NADP}^{+}$ & - & 62 \\
\hline \multicolumn{3}{|l|}{ Average $B$-factors } \\
\hline Protein $\left(\AA^{2}\right)$ & 13.2 & 35.9 \\
\hline Ligand $\left(\AA^{2}\right)$ & - & 53.3 \\
\hline Water $\left(\AA^{2}\right)$ & 16.3 & 47.7 \\
\hline \multicolumn{3}{|l|}{$R M S$ deviations } \\
\hline Bond length $(\AA)$ & 0.006 & 0.010 \\
\hline Bond angle $\left({ }^{\circ}\right)$ & 1.033 & 1.200 \\
\hline \multicolumn{3}{|l|}{ Ramachandran plot } \\
\hline Favored (\%) & 99.4 & 99.5 \\
\hline Allowed (\%) & 0.6 & 0.5 \\
\hline Disallowed (\%) & 0 & 0 \\
\hline
\end{tabular}

Values in parentheses indicate data of the highest resolution shell.

${ }^{\mathrm{a}} R_{\text {merge }}=\sum\left|I_{\mathrm{h}}-<I_{\mathrm{h}}\right\rangle \mid / \sum I_{\mathrm{h}}$, where $I_{\mathrm{h}}$ represents the observed intensity and $\left\langle I_{\mathrm{h}}\right\rangle$ is the average intensity.

${ }^{\mathrm{b}} R=\sum|| F_{\text {obs }}|-| F_{\text {calc }}|| / \sum\left|F_{\text {obs }}\right|$, where $\left|F_{\text {obs }}\right|$ and $\left|F_{\text {calc }}\right|$ represent the observed and calculated structure factor amplitudes, respectively. $R_{\text {free }}$ is calculated for a randomly chosen $5 \%$ of reflections, which were not used during refinement calculations.

changes are mediated by appropriate mechanisms, including amino acid utilization and the use of alternative nutrient sources, as required for survival or virulence (Chaussee et al., 2008; Wood et al., 2009).

To date, crystal structures of several SSADHs have been reported (Ahn et al., 2010; Kim et al., 2009; Langendorf et al., 2010; Park and Rhee, 2013; Yuan et al., 2013; Zheng et al., 2013). However, structural information on bacterial SSADHs remains poorly studied in comparison to other dehydrogenases, with regard to their cofactor preferences and distinct dimeric or tetrameric forms. Thus, more detailed investigations are required for comparison with existing SSADH structures, and to improve our understanding of their metabolic functions. Here, we present the cofactor preference and two crystal structures in an apo-form and in a binary complex with $\mathrm{NADP}^{+}$of gram-positive bacterial SSADH from S. pyogenes (SpSSADH). The analysis of kinetic parameters showed that SpSSADH could be classified as an ortholog of GabD, using NADP ${ }^{+}$as the preferred hydride acceptor. Moreover, both structures showed dimeric conformation, unlike the tetrameric structure exhibited by $E$. coli and human SSADHs. The complex structure of SpSSADH with NADP ${ }^{+}$ showed cofactor discriminative recognition by means of several polar residues, which interacted with the 2'-phosphate group of adenine-side ribose in $\mathrm{NADP}^{+}$. Our results provide kinetic and molecular insightful information regarding the cofactor preference of SpSSADH.

\section{MATERIALS AND METHODS}

Preparation of SpSSADH enzyme

The expression and purification of SpSSADH were performed as previously described (Jang et al., 2012). Briefly, the gene encoding SpSSADH was amplified by PCR from $S$. pyogenes genomic DNA, and the amplified DNA fragment was cloned into the pET$28 \mathrm{a}$ vector (Novagen) with a $\mathrm{His}_{6}$ tag. Then, recombinant plasmids were transformed into $E$. coli BL21 (DE3) strain (Novagen). The expressed SpSSADH enzyme was purified using immobilized metal ion affinity chromatography and size-exclusion chromatography. Sequentially, purified enzyme was concentrated to $26 \mathrm{mg} \mathrm{ml}^{-1}$ and stored at $-80^{\circ} \mathrm{C}$. The enzyme concentration was estimated using the Bradford assay, and the molarity of active enzyme was determined by cysteine titration at the unfolded state using 5,5'-dithiobis(2-nitrobenzoate) (Sigma) (Wright and Viola, 1998). 
Table 2. Steady-state kinetic parameters of SpSSADH

\begin{tabular}{cccccc}
\hline Variable ligand & Fixed substrate & $K_{\mathrm{m}}(\mathrm{mM})$ & $k_{\text {cat }}\left(\mathrm{s}^{-1}\right)$ & $k_{\text {cat }} / K_{\mathrm{m}}\left(\mathrm{M}^{-1} \mathrm{~s}^{-1}\right)$ & $R^{2 *}$ \\
\hline $\mathrm{NAD}^{+}$ & $\mathrm{SSA}(0.5 \mathrm{mM})$ & $0.23 \pm 0.02$ & $1.94 \pm 0.08$ & $9.7 \pm 0.5 \times 10^{3}$ & 0.9952 \\
NADP $^{+}$ & SSA $(0.5 \mathrm{mM})$ & $0.10 \pm 0.01$ & $7.20 \pm 0.12$ & $7.2 \pm 0.3 \times 10^{4}$ & 0.9974 \\
\hline
\end{tabular}

${ }^{*} R^{2}=$ Coefficient of determination

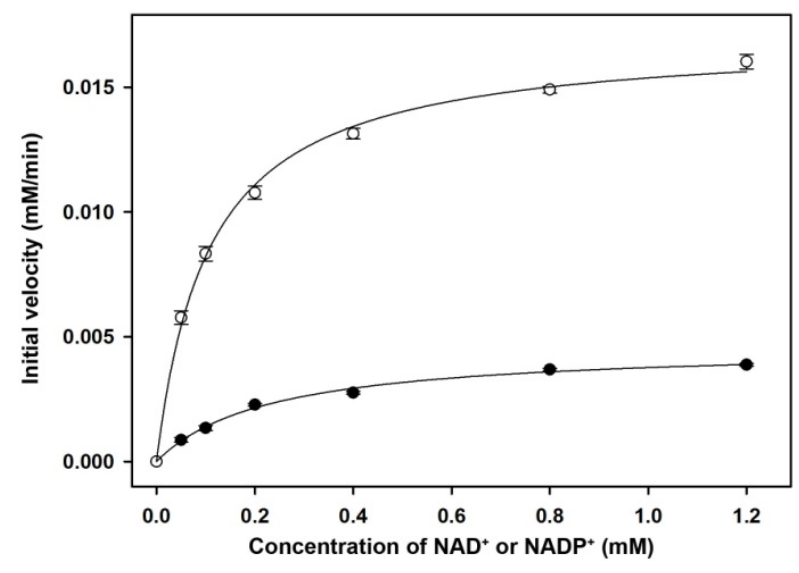

Fig. 1. SpSSADH activity by cofactor preference. Plots of the initial velocity as a function of cofactor concentration are shown for $\mathrm{NAD}^{+}$ (closed circles) and $\mathrm{NADP}^{+}$(open circles). The reaction mixtures containing $0.5 \mathrm{mM} \mathrm{SSA}$ and 0.05-1.2 mM NAD(P) ${ }^{+}$were preincubated at $30^{\circ} \mathrm{C}$ for $5 \mathrm{~min}$. The reaction was initiated by adding of SpSSADH $(1 \mu \mathrm{g} / \mathrm{ml}, 0.0393 \mu \mathrm{M})$.

\section{Enzyme assay for cofactor preference}

The enzyme activities of purified SpSSADH by varying concentrations of both $\mathrm{NAD}^{+}$and $\mathrm{NADP}^{+}$were determined by monitoring the increase of absorbance at UV $340 \mathrm{~nm}\left(\varepsilon_{340} 6.22 \mathrm{mM}^{-1}\right.$ $\mathrm{cm}^{-1}$ ), resulting from the conversion of $\mathrm{NAD}(\mathrm{P})^{+}$to $\mathrm{NAD}(\mathrm{P}) \mathrm{H}$. The reaction mixtures $(1.0 \mathrm{ml})$, containing $20 \mathrm{mM}$ Tris- $\mathrm{HCl}(\mathrm{pH}$ 7.0) and 5.0 mM DTT, were pre-incubated with $0.5 \mathrm{mM} \mathrm{SSA}$ and various concentrations of $\mathrm{NAD}(\mathrm{P})^{+}(0.05-1.2 \mathrm{mM})$ at $30^{\circ} \mathrm{C}$ for 5 $\min$. Then, the reaction was initiated by adding of SpSSADH (1 $\mu \mathrm{g} / \mathrm{ml}, 0.0393 \mu \mathrm{M})$. The initial velocities were estimated from the initial linear portion for the time-courses. All the reactions were performed in triplicate. The kinetic parameters for cofactor preference were determined by nonlinear least-squares fitting to the standard Michaelis-Menten equation and were confirmed with double-reciprocal plots of the initial velocities obtained at varied $\mathrm{NAD}(\mathrm{P})^{+}$concentrations using SigmaPlot (ver. 10.0).

Crystallization and X-ray diffraction data collection Prepared SpSSADH enzyme was crystallized by the hangingdrop vapor diffusion method at $22^{\circ} \mathrm{C}$. Each hanging drop was prepared by mixing $1 \mu$ protein solution and $1 \mu$ reservoir solution, and was equilibrated over a $500 \mu \mathrm{l}$ reservoir solution. The crystals of apo-SpSSADH were obtained in reservoir solution containing 0.1 M MES monohydrate $(\mathrm{pH}$ 5.8-6.1) and 18-23\% (w/v) PEG 4000 (Jang et al., 2012). For co-crystallization with $\mathrm{NADP}^{+}$, the protein solution was mixed with $\mathrm{NADP}^{+}$in a $1: 10$ molar ratio. The crystals of SpSSADH-NADP ${ }^{+}$complex were obtained in a buffer consisting of $0.1 \mathrm{M}$ sodium acetate trihydrate ( $\mathrm{pH} 4.6)$ and $2 \mathrm{M}$ ammonium sulfate.
For cryogenic experiments, both crystals were transferred into a cryoprotection solution consisting of $25 \%(\mathrm{v} / \mathrm{v})$ ethylene glycol in reservoir solution, and were flash-frozen in a stream of nitrogen gas. X-ray diffraction data of SpSSADH crystals in an apoform and in a binary complex with $\mathrm{NADP}^{+}$were collected on a beamline BL26B1 at SPring-8 (Hyogo, Japan) using a Rigaku/MSC Jupiter 210 CCD detector, and on a beamline 5C at the Pohang Light Source (Korea) using an ADSC Quantum 315r CCD detector, respectively. The diffraction data were collected at the resolution of $1.6 \AA$ for apo-crystals, and $2.1 \AA$ for $\mathrm{NADP}^{+}$complex crystals. All data sets were indexed, integrated and scaled using HKL-2000 software package (Otwinowski and Minor, 1997).

\section{Structure determination and refinement}

The crystal structure was solved by the molecular-replacement (MR) method using CNS program (Brunger et al., 1998) with the structure of E. coli SSADH (EcSSADH, PDB ID 3JZ4) (Langendorf et al., 2010) as the search model. The SpSSADH crystals in an apo-form and in a binary complex with NADP ${ }^{+}$ belonged to the orthorhombic $P 2{ }_{1} 2_{1} 2_{1}$ and tetragonal $P 4_{3} 2_{1} 2$ space groups, respectively. The structural refinement was performed with PHENIX program (Adams et al., 2002), and the model was rebuilt with COOT (Emsley and Cowtan, 2004). The final model of the apo-structure contained one dimer, and the final $R_{\text {factor }}$ and $R_{\text {free }}$ values were $17.4 \%$ and $19.9 \%$, respectively. The refined apo-form structure was then used as an initial model to solve the structure of the binary complex. Structural refinements of the binary complex were performed using a rigid body refinement, followed by a simulated annealing refinement. The final model of the $\mathrm{NADP}^{+}$complex structure was determined using CNS program (Brunger et al., 1998), and also contained one dimer with a single $\mathrm{NADP}^{+}$per subunit, and the final $R_{\text {factor }}$ and $R_{\text {free }}$ values were $20.1 \%$ and $22.4 \%$, respectively. Structural validations of both structures were analyzed using PROCHECK program (Laskowski et al., 1993), and no residue was detected in the disallowed region of the Ramachandran plot (Ramachandran et al., 1963). The data collection and final refinement statistics are summarized in Table 1. All structural figures were prepared using PyMOL (Schrodinger, 2010).

\section{Protein data bank accession number}

The atomic coordinates and structure factors of the apo-form and the binary complex structures have been deposited in the Protein Data Bank (http://www.rcsb.org/pdb) with accession numbers $4 \mathrm{OGD}$ and $4 \mathrm{OHT}$, respectively.

\section{RESULTS AND DISCUSSION}

Catalytic properties of SpSSADH by cofactor preference Although SSADHs from various species have been classified as $\mathrm{GabD}$ and Ynel, depending on the cofactor preference that is utilized either $\mathrm{NADP}^{+}$or $\mathrm{NAD}^{+}$, their catalytic efficiencies have shown various differences between each cofactors. In the case of GabD, the EcSSADH has been reported to allow both $\mathrm{NADP}^{+}$ 
Kinetic and Structural Studies on the Cofactor Preference of SpSSADH

Eun Hyuk Jang et al.

A
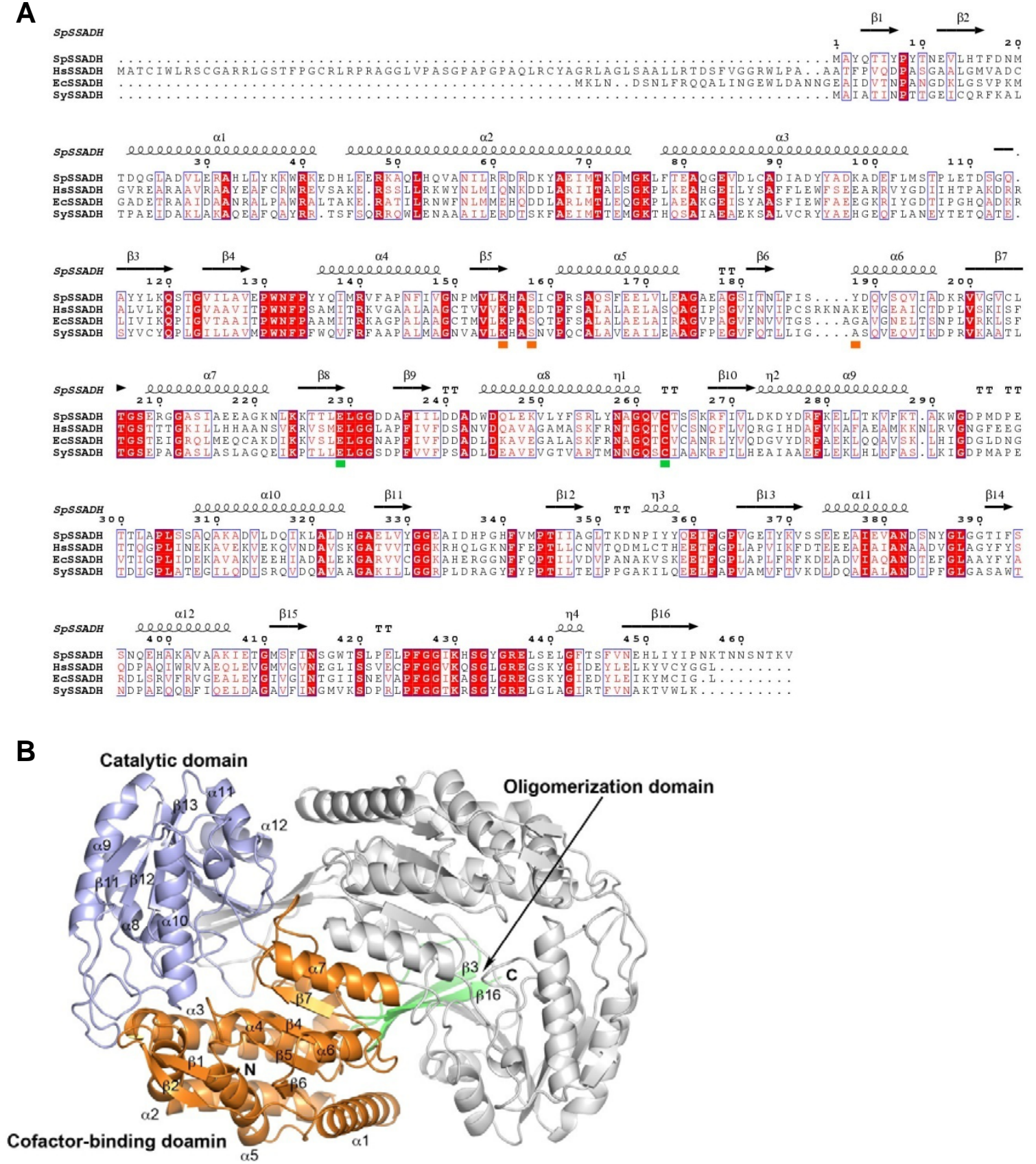

Fig. 2. Sequence alignment and overall structure of SpSSADH. (A) Amino acid sequence alignment of SpSSADH with other SSADHs. SpSSADH, HsSSADH, EcSSADH and SySSADH are representations of Streptococcus pyogenes SSADH (GI: 383493878), human SSADH (GI: 3766467), E. coli SSADH (GI: 120777) and Synechococcus SSADH (GI: 170079362), respectively. Secondary structure elements are drawn on the basis of SpSSADH structure and shown with helices ( $\alpha$-helix) and arrows ( $\beta$-strand) at the top of the aligned sequences. Strictly conserved residues are shown with white characters on a red background, and highly similar residues are shown in red characters with blue frames. The catalytic residues and the residues involved in stabilization of the $2^{\prime}$-phosphate group of adenine-side ribose in NADP ${ }^{+}$are indicated by squares at the bottom of the sequences with green and orange colors, respectively. This figure was prepared using ESPript (Gouet et al., 1999). (B) Overall structure of SpSSADH dimer. The catalytic domain, the cofactor-binding domain and the oligomerization domain of one subunit are colored in blue, orange and green, respectively. Secondary structure elements are labeled on the corresponding sites in the monomeric structure.

and $\mathrm{NAD}^{+}$as cofactors, but its activity with $\mathrm{NADP}^{+}$was more than 20-fold higher than that with $\mathrm{NAD}^{+}$(Jaeger et al., 2008). Moreover, Drosophila melanogaster MalE-DmSSADH showed 7-fold higher activity in the presence of $\mathrm{NAD}^{+}$than $\mathrm{NADP}^{+}$
(Rothacker and Ilg, 2008). On the other hand, in the case of Ynel, the SSADHs from Bacillus subtilis (Park et al., 2014) and Salmonella typhimurium (Zheng et al., 2013) showed differences in affinity for $\mathrm{NAD}^{+}$or $\mathrm{NADP}^{+}$, but their catalytic efficiency ex- 
A

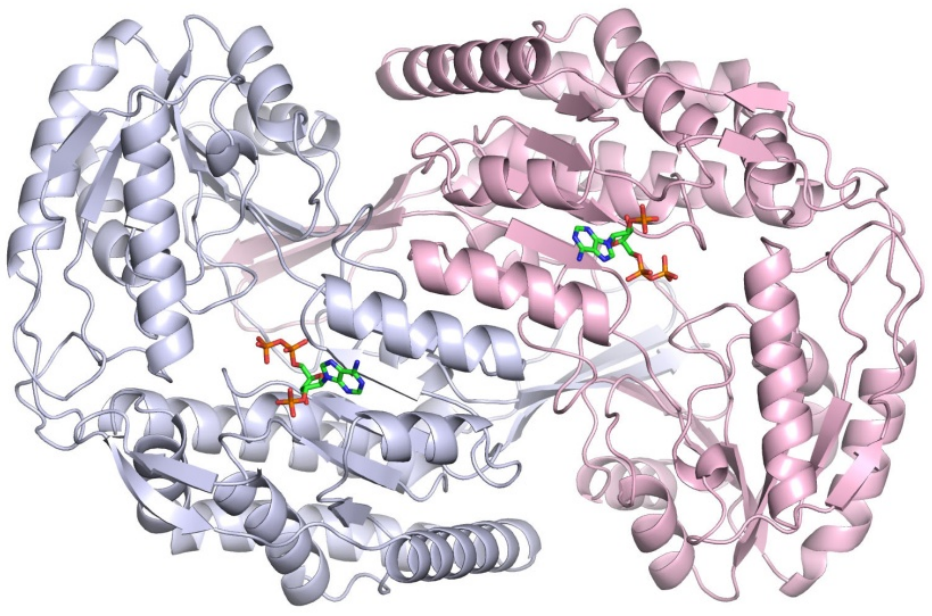

B
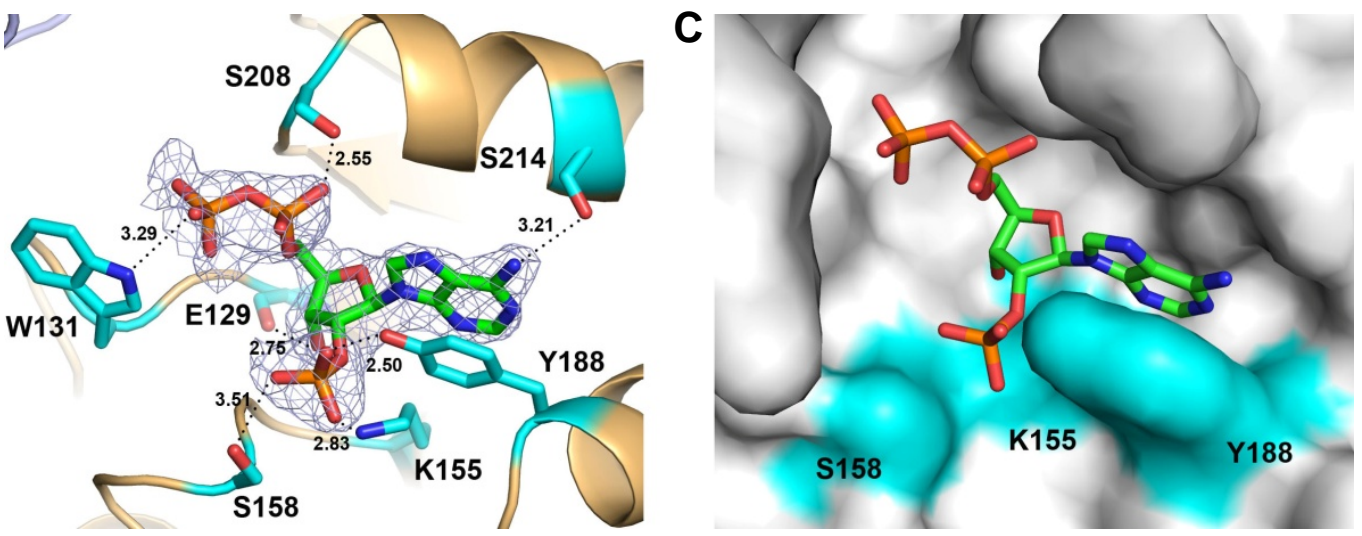

Fig. 3. Binary complex structure of SpSSADH. (A) Overall structure of SpSSADH dimer with partial NADP ${ }^{+}$. Each subunit of the binary complex are shown in blue and pink, respectively. The adenine moiety of $\mathrm{NADP}^{+}$in a form of 2'P-ADP is located at the cofactor-binding domain per each subunit, and represented by stick model in green. (B) Cofactor-binding site of SpSSADH. The NADP binding site of SpSSADH is shown in orange by cartoon model. The SpSSADH residues forming hydrogen bonds with NADP ${ }^{+}$are shown as stick model in cyan. The bound NADP ${ }^{+}$ in the form of 2'P-ADP, is represented by stick model in green with omitted $2 F_{\mathrm{o}}-F_{\mathrm{c}}$ electron density map at $1.0 \sigma$ (blue mesh). The hydrogen bond interactions are displayed with black dotted lines with the distances. (C) Surface model for the cofactor-binding pocket of SpSSADH. The bound $\mathrm{NADP}^{+}$, in the form of 2'P-ADP, is shown in green-colored stick model. The SpSSADH residues involved in stabilization of the 2'phosphate group of ribose in $\mathrm{NADP}^{+}$are highlighted in cyan.

hibited similar values in both cofactors.

To investigate the cofactor preference of SpSSADH, initial velocities were determined by varying the concentration of $\mathrm{NAD}^{+}$or $\mathrm{NADP}^{+}$at a fixed concentration of SSA. The activities of SpSSADH depending on various concentrations of each cofactor followed Michaelis-Menten kinetics (Fig. 1). The $K_{\mathrm{m}}$ and $k_{\text {cat }}$ values for $\mathrm{NADP}^{+}$as the cofactor were approximately 2-fold lower and 4-fold higher than the values for $\mathrm{NAD}^{+}$, respectively (Table 2). As a result, SpSSADH showed more than 7-fold higher values of the catalytic efficiency $\left(k_{\text {cat }} / K_{\mathrm{m}}\right)$ for $\mathrm{NADP}^{+}$than that for $\mathrm{NAD}^{+}$, which were estimated to be $7.2 \pm 0.3 \times 10^{4} \mathrm{M}^{-1} \mathrm{~s}^{-1}$ and $9.7 \pm 0.5 \times 10^{3} \mathrm{M}^{-1} \mathrm{~s}^{-1}$, respectively. These results indicate that SpSSADH prefers $\mathrm{NADP}^{+}$over $\mathrm{NAD}^{+}$, although it can use both cofactors as the hydride acceptor. Thus, SpSSADH can be classified as an ortholog of GabD, corresponding to an $\mathrm{NADP}^{+}$dependent SSADH.

\section{Overall structure of apo-SpSSADH}

The crystal structure of apo-form SpSSADH was determined at the high resolution of $1.6 \AA$ using the MR method, and showed the two subunits in an asymmetric unit with the crystallographic symmetry of $P 2{ }_{1} 2_{1} 2_{1}$ space group representing the functional unit of the homodimer. Consistent with the structural observation, SpSSADH existed as a dimer in solution, which was characterized by size-exclusion chromatography. The PISA server (Krissinel and Henrick, 2007) also predicted a dimeric interface with $5,033 \AA^{2}$ of total solvent-accessible buried surface area for this protein.

Analysis of multiple alignments of SpSSADH with other SSADHs showed a low sequence identity of approximately $35 \%$ (Fig. 2A). Despite this low sequence similarity, monomeric SpSSADH is composed of three domains, similar to previously reported SSADH structures (Ahn et al., 2010; Kim et al., 2009; Langendorf et al., 2010; Park and Rhee, 2013; Yuan et al., 2013; Zheng et al., 2013): the N-terminal cofactor-binding domain (residues 2-103, 122-230 and 423-446), the catalytic domain (residues 231-422) and the oligomerization domain (residues 104121 and 447-456) (Figs. 2A and 2B). Specifically, the catalytic domain consists of a seven-stranded $\beta$-sheet $(\beta 9-\beta 15)$ between two $\alpha$-helices $(\alpha 8, \alpha 9)$ on one side, and three $\alpha$-helices $(\alpha 10$ - 

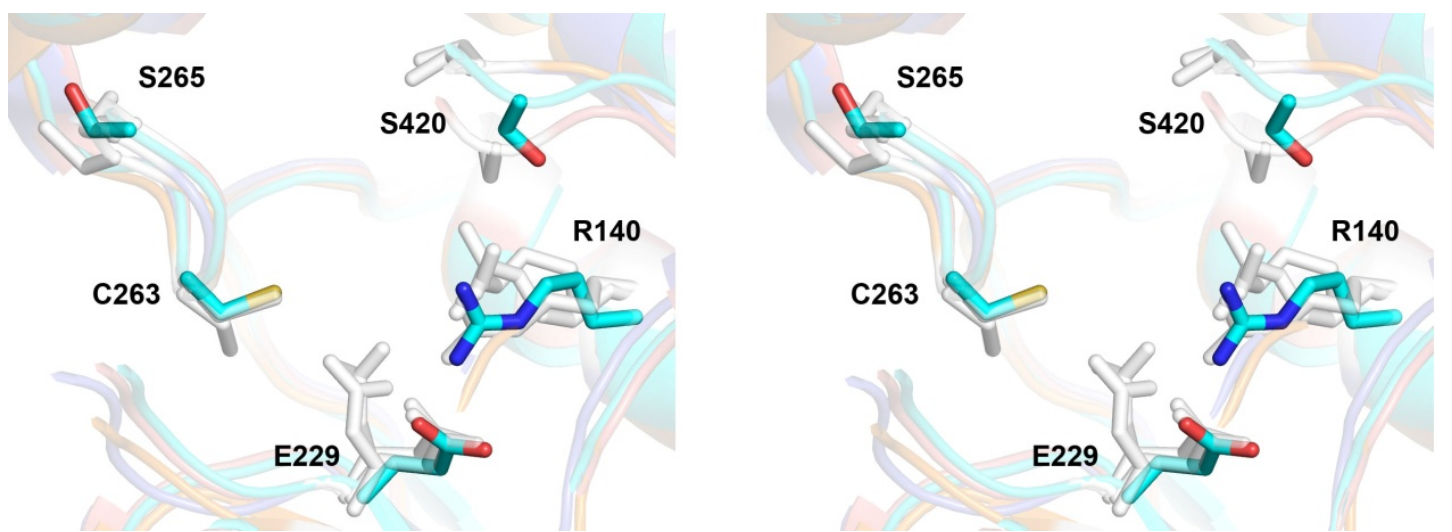

Fig. 4. Stereo view of superimposed structures at the active site. The active site of apo-form SpSSADH structure (cyan) is compared with HsSSADH (PDB ID 2W8O, orange), EcSSADH (PDB ID 3JZ4, blue) and SySSADH (PDB ID 4ITA, pink). The catalytic and conserved residues of SpSSADH are shown as stick model in cyan and labeled in black characters, whereas the corresponding residues of other SSADHs are shown in transparent gray stick model. The Ser265 residue of SpSSADH is shown at the position of the partner cysteine residue in HsSSADH (Cys342), which forms a disulfide bond with the catalytic cysteine.

$\alpha 12)$ on the other side. The cofactor-binding domain displays two Rossmann folds, with a combination of N-terminal two antiparallel $\beta$-strands $(\beta 1, \beta 2)$ and a central five-stranded parallel $\beta$ sheet $(\beta 4-\beta 8)$, surrounded by three $\alpha$-helices $(\alpha 1, \alpha 6, \alpha 7)$ and four $\alpha$-helices $(\alpha 2-\alpha 5)$. Two antiparallel $\beta$-strands $(\beta 3, \beta 16)$ constructing the oligomerization domain are stretched across the center of the dimer interface. The catalytic residues, which have been characterized in other SSADHs (Ahn et al., 2010; Kim et al., 2009; Langendorf et al., 2010; Park and Rhee, 2013; Yuan et al., 2013; Zheng et al., 2013) are conserved in SpSSADH (Cys263 and Glu229) and are located in the middle of the catalytic and cofactor-binding domains. Cys263, as a nucleophilic attacker, is located in the catalytic loop between $\alpha 8$ and $\beta 10$ of the catalytic domain, and Glu229, as a general base, is located at the end of $\beta 8$.

\section{Structure of SpSSADH in a binary complex with NADP ${ }^{+}$}

To confirm the structural basis for cofactor preference and binding properties of SpSSADH, the binary complex structure of SpSSADH with $\mathrm{NADP}^{+}$was also determined at a resolution of $2.1 \AA$ (Fig. $3 A$ ). The overall structure of the binary complex was very similar to the apo-structure, with a root mean square deviation of $0.73 \AA$. In the binary complex structure of SpSSADH, the adenine moiety of $\mathrm{NADP}^{+}$molecule was determined to be in the form of 2'-monophosphoadenosine-5'-diphosphate (2'P-ADP, PubChem ID 165230), using a well-defined electron density map (Fig. 3B). However, the nicotinamide ring portion of $\mathrm{NADP}^{+}$could not be modeled, due to the absence of electron density. Similar phenomena that the nicotinamide ring and linked ribose portions of the cofactor are flexible enough to generate a poor electron density map have been reported in human SSADH (HsSSADH) and other ALDHs (Di Costanzo et al., 2007; Kim et al., 2009).

The partial $\mathrm{NADP}^{+}$in the binary complex is located between $\alpha 6$ and $\alpha 7$ in the cofactor-binding domain, and is stabilized by hydrogen bonds with neighboring residues (Fig. 3B). The nitrogen atom on the edge of the adenine base is hydrogen bonded with the side chain of Ser214. The 2'-phosphate group of ribose is stabilized by hydrogen bonds with the side chains of Lys155, Ser158 and Tyr188. In particular, three residues are considered to contribute to the cofactor selection of $\mathrm{NADP}^{+}$(Fig. 3C). The main chain of Glu129 forms a hydrogen bond with the $3^{\prime}$-hydroxyl group of ribose. The pyrophosphate group of the cofactor maintains only a few hydrogen bonds with the side chains of Trp131 and Ser208. In addition to the hydrogen bond forming residues, the adenine base is inserted into a hydrophobic pocket formed by several residues, including Val128, Val191, Leu205 and lle215.

\section{Structural comparison of SpSSADH with other SSADHs}

Structural comparison of SpSSADH was performed by superimposition with the structures of SSADHs from human (Kim et al., 2009), E. coli (Ahn et al., 2010; Langendorf et al., 2010) and Synechococcus (SySSADH) (Park and Rhee, 2013; Yuan et al., 2013). Although the monomeric structure of SpSSADH was quite similar to other SSADHs involving the cofactor- and substrate-binding sites, SpSSADH showed a dimeric interface, unlike the tetrameric structure exhibited by EcSSADH and $\mathrm{HsSSADH}$. Another significant feature of SpSSADH is the presence of a single cysteine residue (Cys263) at the catalytic loop (Fig. 4), which is similar to most members of the ALDH family (Park and Rhee, 2013). HsSSADH contains paired cysteine residues (Cys340 and Cys342) for disulfide bond formation, but the position of the partner cysteine in SpSSADH is replaced by Ser265. Thus, redox-switch modulation proposed in HsSSADH is not applicable in SpSSADH. Moreover, the active site of SpSSADH contains the catalytic residues (Cys263 and Glu229) and several conserved residues (Arg140 and Ser420) that are involved in substrate stabilization in other SSADHs.

For structure-based confirmation of the cofactor preference, the binding of $\mathrm{NADP}^{+}$in the binary complex of SpSSADH was compared with the structures of HsSSADH (Fig. 5A) and SySSADH (Fig. 5B) in complexes with their cofactors. Although the binary complex structure of SpSSADH contained a partial $\mathrm{NADP}^{+}$, the structural superimposition of SpSSADH with other SSADHs revealed that the adenine moiety of $\mathrm{NADP}^{+}$was located at almost the identical position of the cofactor-binding site. In addition, the residues forming hydrogen bonds with the adenine moiety of $\mathrm{NADP}^{+}$in SpSSADH are mostly conserved in other SSADHs, and located at similar positions to corresponding residues. Previous studies on the structure of SySSADH have suggested that the particular residue in the cofactor-binding site plays an important role in selective recognition of the cofactor (Park and Rhee, 2013; Yuan et al., 2013). For example, a polar residue with short side chain, such as serine or threonine, in the cofactor-binding pocket permits $\mathrm{NADP}^{+}$rather than $\mathrm{NAD}^{+}$, whereas a residue with negative-charged long side chain, such 

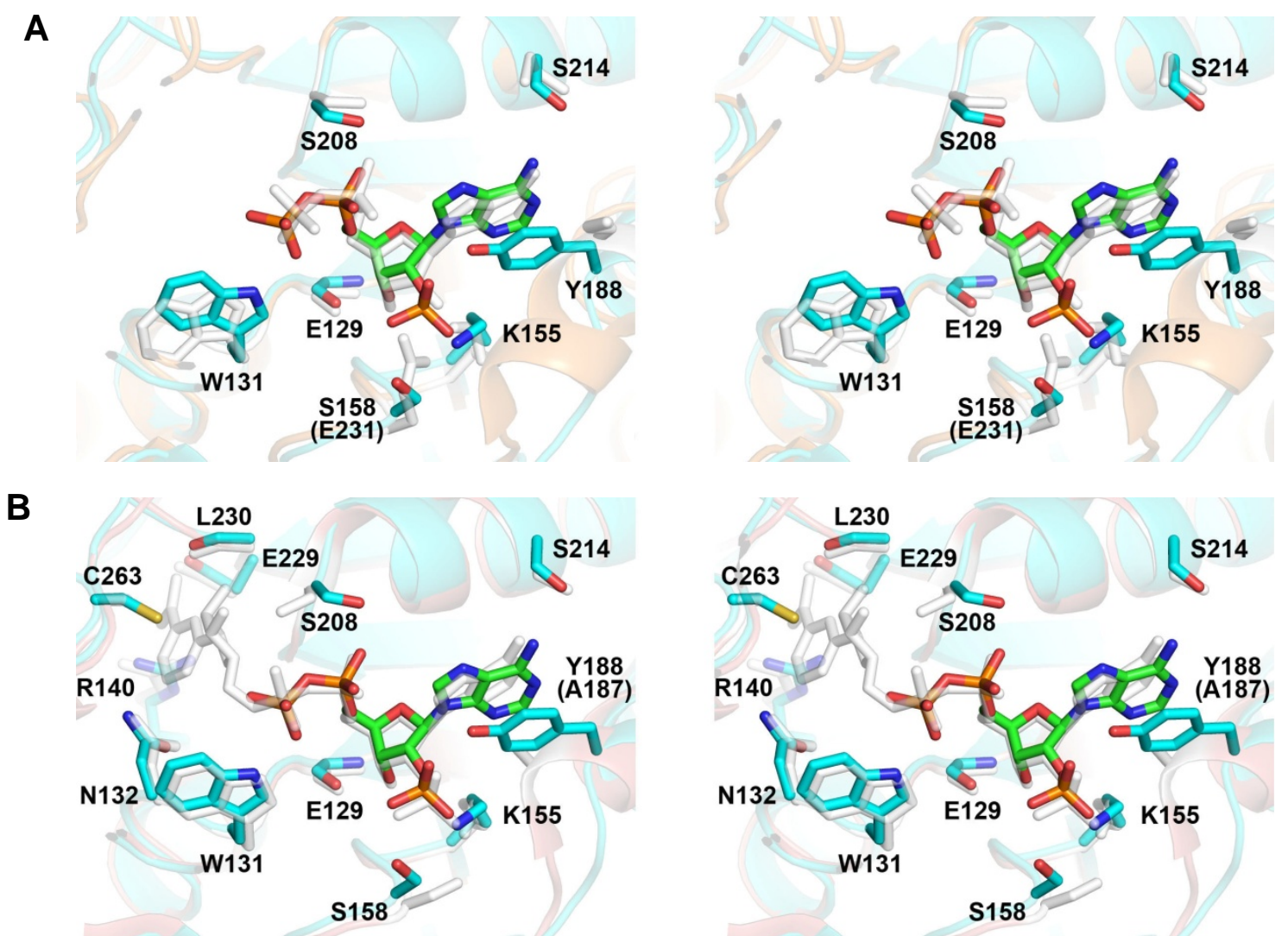

Fig. 5. Structural comparison of SpSSADH at the cofactor-binding site. (A) Stereo view of the superimposition of SpSSADH binary complex with $\mathrm{NAD}^{+}$-binding HsSSADH structure (PDB ID 2W8R). Superimposed structures of SpSSADH and HsSSADH are shown in cyan- and orangecolored cartoon model, respectively. The partial NADP ${ }^{+}\left(2^{\prime} \mathrm{P}-\mathrm{ADP}\right)$ bound to SpSSADH is shown as stick model in green, and the ADP moiety of $\mathrm{NAD}^{+}$in HsSSADH structure is shown in transparent gray. The residues of SpSSADH involved in the stabilization of NADP ${ }^{+}$are shown in cyan by stick model, whereas the corresponding residues of HsSSADH are shown in transparent gray. The residues with black characters are labeled on the basis of SpSSADH. The residue of HsSSADH (Glu231) corresponding to the cofactor preference determining residue is labeled in parentheses. (B) Stereo view of superimposition of the SpSSADH binary complex with the NADP ${ }^{+}$-binding SySSADH structure (PDB ID 3VZ3). Superimposed structures of SpSSADH and SySSADH are shown in cyan- and pink-colored cartoon model, respectively. The partial NADP ${ }^{+}$ (2'P-ADP) bound to SpSSADH is shown in green by stick model, and the NADP ${ }^{+}$in the SySSADH structure is shown in transparent gray. The residues of SpSSADH, which are conserved in SySSADH and involved in cofactor stabilization, are shown in cyan-colored stick model, whereas the corresponding residues of SySSADH are represented in transparent gray. The residues with black characters are labeled on the basis of SpSSADH. The residue of SySSADH (Ala187) corresponding to Tyr188 of SpSSADH, which is involved in the stabilization of the 2'-phosphate group of adenine-side ribose in $\mathrm{NADP}^{+}$, is labeled in parentheses.

as glutamate, prefers $\mathrm{NAD}^{+}$(Yuan et al., 2013). Interestingly, the binary complex structure of SpSSADH with $\mathrm{NADP}^{+}$shows that Ser158 is located at the critical position for cofactor recognition, while HsSSADH contains Glu231 residue in this position (Fig. $5 A)$. This result implies that SpSSADH prefers $\mathrm{NADP}^{+}$rather than $\mathrm{NAD}^{+}$as a cofactor, which is consistent with the NADP ${ }^{+}$ binding SSADHs from E. coli and Synechococcus containing Ser182 and Ser157, respectively.

In the different structural features inferring the NADP ${ }^{+}$-binding properties of SpSSADH (Fig. 5B), the 2'-phosphate group of adenine-side ribose in $\mathrm{NADP}^{+}$is further stabilized by hydrogen bond with the side chain of Tyr188, which is not found in EcSSADH (Gly213) or SySSADH (Ala187). As shown in Fig. 3B, the hydrogen bond in the corresponding interaction with Tyr188 $(2.50 \AA)$ is formed at a closer distance than that of Ser158 (3.51 $\AA$ ), which is the key residue for the cofactor discrimination. Additionally, the value of $K_{m}$ for $\operatorname{NADP}^{+}(0.10 \pm 0.01 \mathrm{mM})$ of SpSSADH was approximately 2 -fold lower than that for $\mathrm{NAD}^{+}$
$(0.23 \pm 0.02 \mathrm{mM})$, as described in the analysis of kinetic parameters, and was also 4-fold lower than that for NADP ${ }^{+}$of SySSADH (0.44 mM) (Park and Rhee, 2013). Thus, we assume that Tyr188 of SpSSADH may contribute not only facilitating the corresponding binding of either $\mathrm{NADP}^{+}$or $\mathrm{NAD}^{+}$, but also enhancing the binding affinity of $\mathrm{NADP}^{+}$particularly, although further studies remain to elucidate its function. Apart from this, the residues, Asn132, Arg140 and Leu230, located at the entrance of the active site in SpSSADH, are conserved as the cofactorbinding residues forming hydrogen bonds with the nicotinamide moiety of $\mathrm{NADP}^{+}$in other SSADHs.

\section{ACKNOWLEDGMENTS}

We thank the staff of beamline $5 \mathrm{C}$ at Pohang Accelerator Laboratory in South Korea, and of beamline BL26B1 at SPring-8 in Japan for assistance during $\mathrm{X}$-ray data collection. This work was supported by a Korea University Grant 2013 (to Y. M. Chi). 


\section{REFERENCES}

Adams, P.D., Grosse-Kunstleve, R.W., Hung, L.W., loerger, T.R., McCoy, A.J., Moriarty, N.W., Read, R.J., Sacchettini, J.C., Sauter, N.K., and Terwilliger, T.C. (2002). PHENIX: building new software for automated crystallographic structure determination. Acta Crystallogr. D Biol. Crystallogr. 58, 1948-1954.

Ahn, J.W., Kim, Y.G., and Kim, K.J. (2010). Crystal structure of nonredox regulated SSADH from Escherichia coli. Biochem. Biophys. Res. Commun. 392, 106-111.

Bouche, N., and Fromm, H. (2004). GABA in plants: just a metabolite? Trends Plant Sci. 9, 110-115.

Bouche, N., Fait, A., Bouchez, D., Moller, S.G., and Fromm, H. (2003). Mitochondrial succinic-semialdehyde dehydrogenase of the gamma-aminobutyrate shunt is required to restrict levels of reactive oxygen intermediates in plants. Proc. Natl. Acad. Sci. USA 100, 6843-6848.

Brunger, A.T., Adams, P.D., Clore, G.M., DeLano, W.L., Gros, P., Grosse-Kunstleve, R.W., Jiang, J.S., Kuszewski, J., Nilges, M., Pannu, N.S., et al. (1998). Crystallography \& NMR system: A new software suite for macromolecular structure determination. Acta Crystallogr. D Biol. Crystallogr. 54, 905-921.

Chaussee, M.A., Callegari, E.A., and Chaussee, M.S. (2004). Rgg regulates growth phase-dependent expression of proteins associated with secondary metabolism and stress in Streptococcus pyogenes. J. Bacteriol. 186, 7091-7099.

Chaussee, M.A., Dmitriev, A.V., Callegari, E.A., and Chaussee, M.S. (2008). Growth phase-associated changes in the transcriptome and proteome of Streptococcus pyogenes. Arch. Microbiol. 189, 27-41.

Cunningham, M.W. (2000). Pathogenesis of group A streptococcal infections. Clin. Microbiol. Rev. 13, 470-511.

de Carvalho, L.P., Ling, Y., Shen, C., Warren, J.D., and Rhee, K.Y (2011). On the chemical mechanism of succinic semialdehyde dehydrogenase (GabD1) from Mycobacterium tuberculosis. Arch Biochem. Biophys. 509, 90-99.

Di Costanzo, L., Gomez, G.A., and Christianson, D.W. (2007) Crystal structure of lactaldehyde dehydrogenase from Escherichia coli and inferences regarding substrate and cofactor specificity. J. Mol. Biol. 366, 481-493.

Emsley, P., and Cowtan, K. (2004). Coot: model-building tools for molecular graphics. Acta Crystallogr. D Biol. Crystallogr. 60, 2126-2132.

Fait, A., Fromm, H., Walter, D., Galili, G., and Fernie, A.R. (2008). Highway or byway: the metabolic role of the GABA shunt in plants. Trends Plant Sci. 13, 14-19.

Fuhrer, T., Chen, L., Sauer, U., and Vitkup, D. (2007). Computational prediction and experimental verification of the gene encoding the $\mathrm{NAD}^{+} / \mathrm{NADP}^{+}$-dependent succinate semialdehyde dehydrogenase in Escherichia coli. J. Bacteriol. 189, 8073-8078.

Gouet, P., Courcelle, E., Stuart, D.I., and Metoz, F. (1999). ESPript: analysis of multiple sequence alignments in PostScript. Bioinformatics 15, 305-308.

Grant, S.S., and Hung, D.T. (2013). Persistent bacterial infections, antibiotic tolerance, and the oxidative stress response. Virulence 4, 273-283.

Jaeger, M., Rothacker, B., and Ilg, T. (2008). Saturation transfer difference NMR studies on substrates and inhibitors of succinic semialdehyde dehydrogenases. Biochem. Biophys. Res. Commun. 372, 400-406.

Jang, E.H., Lim, J.E., Chi, Y.M., and Lee, K.S. (2012). Crystallization and preliminary $\mathrm{X}$-ray crystallographic studies of succinic semialdehyde dehydrogenase from Streptococcus pyogenes. Acta Crystallogr. Sect. F Struct. Biol. Cryst. Commun. 68, 288291

Kim, Y.G., Lee, S., Kwon, O.S., Park, S.Y., Lee, S.J., Park, B.J., and
Kim, K.J. (2009). Redox-switch modulation of human SSADH by dynamic catalytic loop. EMBO J. 28, 959-968.

Kim, K.J., Pearl, P.L., Jensen, K., Snead, O.C., Malaspina, P., Jakobs, C., and Gibson, K.M. (2011). Succinic semialdehyde dehydrogenase: biochemical-molecular-clinical disease mechanisms, redox regulation, and functional significance. Antioxid. Redox. Signal. $15,691-718$

Krissinel, E., and Henrick, K. (2007). Inference of macromolecular assemblies from crystalline state. J. Mol. Biol. 372, 774-797.

Langendorf, C.G., Key, T.L., Fenalti, G., Kan, W.T., Buckle, A.M., Caradoc-Davies, T., Tuck, K.L., Law, R.H., and Whisstock, J.C. (2010). The X-ray crystal structure of Escherichia coli succinic semialdehyde dehydrogenase; structural insights into NADP $/$ enzyme interactions. PLoS One 5, e9280.

Laskowski, R.A., MacArthur, M.W., Moss, D.S., and Thornton, J.M. (1993). PROCHECK: a program to check the stereochemical quality of protein structures. J. Appl. Crystalogr. 26, 283-291.

Otwinowski, Z., and Minor, W. (1997). Processing of X-ray diffraction data collected in oscillation mode. Methods Enzymol. 276, 307326.

Park, J., and Rhee, S. (2013). Structural basis for a cofactordependent oxidation protection and catalysis of cyanobacterial succinic semialdehyde dehydrogenase. J. Biol. Chem. 288, 15760-15770.

Park, S.A., Park, Y.S., and Lee, K.S. (2014). Kinetic characterization and molecular modeling of $N A D(P)^{+}$-dependent succinic semialdehyde dehydrogenase from Bacillus subtilis as an ortholog Ynel. J. Microbiol. Biotechnol. 24, 954-958.

Ralph, A.P., and Carapetis, J.R. (2013). Group a streptococcal diseases and their global burden. Curr. Top. Microbiol. Immunol. $368,1-27$

Ramachandran, G.N., Ramakrishnan, C., and Sasisekharan, V. (1963). Stereochemistry of polypeptide chain configurations. J. Mol. Biol. 7, 95-99.

Rothacker, B., and Ilg, T. (2008). Functional characterization of a Drosophila melanogaster succinic semialdehyde dehydrogenase and a non-specific aldehyde dehydrogenase. Insect Biochem. Mol. Biol. 38, 354-366.

Schneider, B.L., Ruback, S., Kiupakis, A.K., Kasbarian, H., Pybus, C., and Reitzer, L. (2002). The Escherichia coli gabDTPC operon: specific gamma-aminobutyrate catabolism and nonspecific induction. J. Bacteriol. 184, 6976-6986

Schrodinger, L.L.C. (2010). The PyMOL Molecular Graphics System, Version $1.3 \mathrm{r} 1$

Trainor, V.C., Udy, R.K., Bremer, P.J., and Cook, G.M. (1999). Survival of Streptococcus pyogenes under stress and starvation. FEMS Microbiol. Lett. 176, 421-428.

Wood, D.N., Chaussee, M.A., Chaussee, M.S., and Buttaro, B.A. (2005). Persistence of Streptococcus pyogenes in stationaryphase cultures. J. Bacteriol. 187, 3319-3328.

Wood, D.N., Weinstein, K.E., Podbielski, A., Kreikemeyer, B., Gaughan, J.P., Valentine, S., and Buttaro, B.A. (2009). Generation of metabolically diverse strains of Streptococcus pyogenes during survival in stationary phase. J. Bacteriol. 191, $6242-6252$.

Wright, S.K., and Viola, R.E. (1998). Evaluation of methods for the quantitation of cysteines in proteins. Anal. Biochem. 265, 8-14.

Yuan, Z., Yin, B., Wei, D., and Yuan, Y.R. (2013). Structural basis for cofactor and substrate selection by cyanobacterium succinic semialdehyde dehydrogenase. J. Struct. Biol. 182, 125-135.

Zheng, H., Beliavsky, A., Tchigvintsev, A., Brunzelle, J.S., Brown, G., Flick, R., Evdokimova, E., Wawrzak, Z., Mahadevan, R., Anderson, W.F., et al. (2013). Structure and activity of the $\mathrm{NAD}(\mathrm{P})^{+}$-dependent succinate semialdehyde dehydrogenase Ynel from Salmonella typhimurium. Proteins 81, 1031-1041. 